\title{
A Detailed Analysis of the Quantum Relativistic Motion of an Electron
}

\author{
Gregory L. Light ${ }^{1}$ \\ ${ }^{1}$ Department of Management, Providence College, 1 Cunningham Square, Providence, Rhode Island, USA \\ Correspondence: Gregory L. Light, Department of Management, Providence College, 1 Cunningham Square, \\ Providence, Rhode Island 02918, USA. Tel: 1-401-865-2662. E-mail: glight@providence.edu
}

Received: August 21, 2012 Accepted: September 10, 2012 Online Published: November 1, 2012

doi:10.5539/apr.v5n1p7

URL: http://dx.doi.org/10.5539/apr.v5n1p7

\begin{abstract}
Based on Pauli spin matrices, this paper settles the geometry of our previously constructed combined spacetime 4-manifold to be $\{(\mathrm{t}+\mathrm{ti}, \mathrm{x}+\mathrm{yi}, \mathrm{y}+\mathrm{zi}, \mathrm{z}+\mathrm{xi})$ : $(\mathrm{t}, \mathrm{x}, \mathrm{y}, \mathrm{z})$ of Minkowski space $\}$ locally. Thereby we specify the motions of the two eigenvectors of the spin operator and show that they satisfy Dirac equation of a free electron; accordingly, an electron is a point particle born out of a spinning classic electric field with each of its energy density points rotating along two perpendicularly connected semi-circles. We also demonstrate the pair-annihilation process via a simple trigonometric identity, and we conclude with some pertinent remarks.
\end{abstract}

Keywords: spinors, positron, pair-annihilation, spin $1 \frac{1}{2}$, particle-wave duality

\section{Introduction}

This paper continues on our previous hypothesis of a "combined spacetime 4-manifold" (Light, 2012):

$$
M^{[3]}:=\left\{\left(p^{[1]}, p^{[2]}\right) \in M^{[1]} \times B, B \subset M^{[2]} \mid h\left(p^{[1]}\right)=p^{[2]}, h=\text { a diffeomorphism }\right\},
$$

where $\mathrm{M}^{[1]}$ is the universe of particles and $\mathrm{B}$ is a macroscopic black hole that is contained in $\mathfrak{M}^{[2]}$ - - a universe of electromagnetic waves. Here we specify that the two copies of the universes coincide locally as

$$
M^{[3]}=\left\{\left(\begin{array}{c}
t+t i \\
x+y i \\
y+z i \\
z+x i
\end{array}\right) \in \mathbb{C}^{4} \mid(t, x, y, z) \in \mathbb{R}^{1+3} \equiv \text { Minkowski space }\right\}
$$

(i.e., $\mathrm{h}(\mathrm{x}, \mathrm{y}, \mathrm{z})=(\mathrm{z}, \mathrm{x}, \mathrm{y}))$ by a consideration of the following isomorphic relation in a tangent space

$$
T_{p} \mathfrak{M}^{[3]} \text { of a point } p \in \mathfrak{M}^{[3]} \text {. }
$$

First we have

$$
(a+b i)(c+d i)=a c-b d+(b c+a d) i \cong\left(\begin{array}{l}
a c-b d \\
b c+a d
\end{array}\right)=\left(\begin{array}{cc}
a & -b \\
b & a
\end{array}\right)\left(\begin{array}{l}
c \\
d
\end{array}\right) .
$$

As such, we have

$$
i \frac{\partial}{\partial x} \equiv i e_{x} \cong\left(\begin{array}{cc}
0 & -1 \\
1 & 0
\end{array}\right)_{(x, y)}\left(\begin{array}{l}
1 \\
0
\end{array}\right)_{(x, y)}=\left(\begin{array}{l}
0 \\
1
\end{array}\right)_{(x, y)} \cong \frac{\partial}{\partial y} \equiv e_{y} .
$$


Similarly, we then have

$$
i \frac{\partial}{\partial y} \equiv i e_{y} \equiv e_{z} \quad \text { and } \quad i \frac{\partial}{\partial z} \equiv i e_{z} \equiv e_{x} .
$$

As an illustration, consider the vector

$$
v_{p}=\left(\begin{array}{c}
3+5 i \\
5+7 i \\
7+3 i
\end{array}\right) \in T_{p} M^{[3]} \subset \mathbb{C}^{3} .
$$

$v_{p}$ can be represented by a vector at point $\mathrm{p}$ of $\mathfrak{M}^{[3]}$ as

$$
\operatorname{Re}\left(v_{p}\right)=3 e_{x}+5 e_{y}+7 e_{z} \in \mathbb{R}_{(x, y, z)}
$$

that coincides with the vector

$$
\left(3 e_{z}+5 e_{x}+7 e_{y}\right) i \in i \mathbb{R}_{(z, x, y)} \text {. }
$$

That is,

$i \mathbb{R}_{(z, x, y)}^{3}$ results from a $90^{\circ}$-counterclockwise rotation of $\mathbb{R}_{(x, y, z)}^{3}$, and $i \mathbb{R}_{(z, x, y)}^{3}$ coincides with $\mathbb{R}_{(x, y, z)}^{3}$.

To summarize, we have the following identifications between the two sets of basis vectors:

$$
e_{x} \equiv i e_{z}, \quad e_{y} \equiv i e_{x}, \quad \text { and } \quad e_{z} \equiv i e_{y} .
$$

Since Equations in (1.9) are based on our original hypothesis of a combined spacetime 4-manifold, we shall refer to Equations (1.9) as our Assumption 1 (of the three assumptions that will lead our analysis in the sequel; the other two assumptions will likewise be specially noted). In passing, we also note the following three projective planes:

$$
\mathbb{R}_{(x, y)}^{2} \cong \mathbb{C}_{x}, \quad \mathbb{R}_{(y, z)}^{2} \cong \mathbb{C}_{y}, \quad \text { and } \quad \mathbb{R}_{(z, x)} \cong \mathbb{C}_{z}
$$

As for $t i$, we have the postulate of Einstein special relativity,

$$
c^{2}(i t)^{2}-(i x)^{2}-(i y)^{2}-(i z)^{2}=0 .
$$

With the above identities, Pauli spin matrices describe the dynamics of the "electron-wave" as follows:

First,

$$
\left(\begin{array}{ll}
0 & 1 \\
1 & 0
\end{array}\right)_{(x, y)}
$$

indicates the directions of the momenta of the two eigenvectors of the spin operator, $\Lambda(\mathrm{A})$ and $\Lambda(\mathrm{B})$, with $\Lambda(\mathrm{A})$ moving from

$$
\left(\begin{array}{c}
-1 \\
0 \\
0
\end{array}\right) \equiv " 9-o^{\prime} \text { clock" } \equiv W \quad \text { to }\left(\begin{array}{l}
0 \\
1 \\
0
\end{array}\right) \equiv " 12-o^{\prime} \text { clock" } \equiv N
$$


and $\Lambda(\mathrm{B})$ moving from

$$
\left(\begin{array}{l}
0 \\
0 \\
1
\end{array}\right) \equiv T(" t o p ") \quad \text { to }\left(\begin{array}{l}
1 \\
0 \\
0
\end{array}\right) \equiv " 3-o^{\prime} \text { clock" } \equiv E \text {. }
$$

Second,

$$
\left(\begin{array}{cc}
1 & 0 \\
0 & -1
\end{array}\right)_{(x, z)}
$$

indicates that $\Lambda(A)$ is moving from " $12 \equiv N$ " to " $3 \equiv E$ "

and $\Lambda(B)$, from " $3 \equiv E$ " to

$$
\left(\begin{array}{c}
0 \\
0 \\
-1
\end{array}\right) \equiv B(" \text { bottom") }
$$

Third, by Equations in (1.9), we have

$$
\left(\begin{array}{cc}
0 & -i \\
i & 0
\end{array}\right)_{(x, y)} \equiv\left(i e_{y},-i e_{x}\right) \equiv\left(e_{z},-e_{y}\right) \equiv\left(\begin{array}{cc}
0 & 0 \\
0 & -1 \\
1 & 0
\end{array}\right)_{(x, y, z)}
$$

which indicates that $\Lambda(\mathrm{A})$ is moving from " $3 \equiv \mathrm{E}$ " to $\mathrm{T}$

and $\Lambda(B)$, from bottom to

$$
\left(\begin{array}{c}
0 \\
-1 \\
0
\end{array}\right) \equiv " 6 \equiv S
$$

In summary, $\Lambda(\mathrm{A})$ has the motion:

$$
9 \equiv W \rightarrow 12 \equiv N \rightarrow 3 \equiv E \rightarrow T \rightarrow 9 \equiv W
$$

and $\Lambda(\mathrm{B})$,

$$
T \rightarrow 3 \equiv E \rightarrow B \rightarrow 6 \equiv S \rightarrow T .
$$

In short, either eigenvector runs around two perpendicularly connected semi-circles, with the matrix

$$
\left(\begin{array}{cc}
0 & -i \\
i & 0
\end{array}\right)_{(x, y)}
$$

indicating a change of the planes of rotation.

The existing research about our topic largely revolves around intensified studies of the Clifford algebra, especially in connection with the quantum information theories, and of projective geometries of abstract higher-dimensions (Rau, 2009). These pursuits are founded upon the probability interpretation of the quantum wave functions so that they easily involve dimensions more than four (Barut\& Bracken, 1981), devoid of the physical contents of energies. In our view, the waves are electromagnetic waves in $\mathrm{B} \mathrm{M}^{[2]}$; they are naturally entangled by virtue of the geometry of quotient spaces in B. Furthermore, if our geometry of a particle at $(t, x, y$, $\mathrm{z}$ ) in $\mathfrak{M}^{[1]}$ carrying its wave energy around (it, iz, ix, iy) in $\mathrm{B} \subset \mathfrak{M}^{[2]}$ is correct, then the misspecified geometries will likely hamper the development of the technologies sought for (Costa et al., 2012, for a shared interest in 
modeling the Dirac electron physically) .

In the next Section 2 we shall present a detailed analysis to show that our description of an electron satisfies Dirac's equation of a free electron. We distinguish our study from many others by focusing on the motion of the electric field $\mathbb{E} \subset \mathrm{B} \subset \mathrm{M}^{[2]}$ that is responsible for revealing the point particle electron in $\mathfrak{M}^{[1]}$ (cf. Demikhovskii et al., 2010) for a similarly motivated study), not the dynamics of an electron that is subject to an external field (Ahrens et al., 2012).Then we will also show how a pair of electron and positron annihilate each other. In this connection, we break away from "the Dirac's sea of electrons with negative energies" (which was based on the premise of pair-creations out of a vacuum;see, e.g.,a recent paper by (Dreisow et al., 2012) for a continued dependence on this view, but also cf. (Witten, 1981) for the positive energy theorem). "Vacuum" in our model is simply the $\mathrm{B} \subset \mathrm{M}^{[2]}$. In short, our work in this paper replaces the idea of intrinsic spins (which is an inevitable result from the probability interpretation of the quantum waves) with physical spinning motions of waves of (positive) energies in a four-dimensional spacetime.Finally in Section 3 we will close with some pertinent summary remarks.

\section{Analysis}

\subsection{The Spinning Motion of an Electron-Wave}

It is a known fact that the spinning states of an electron are physically separated by 180 degrees. As such, to represent the two eigenvectors $\Lambda(\mathrm{A})$ and $\Lambda(\mathrm{B})$ of the spin operator as two orthogonal vectors for an application of Pauli matrices, one might be led to a contraction of the 360-degree-plane into half of which, i.e., to set up

$$
\hat{\theta} \equiv \frac{1}{2} \theta
$$

so that a physical separation of $\Lambda(\mathrm{A})$ and $\Lambda(\mathrm{B})$ by $\theta=180^{\circ}$ would be displayed as

$$
\hat{\theta}=90^{\circ} \text {. }
$$

However, $\theta$ is a relativistic invariant and the treatment of

$$
\frac{1}{2} \theta=\frac{1}{2} \omega t
$$

would run into the need of a constant reminder of the relationship between the physical $\theta=180^{\circ}$ and the mathematical $\theta$-hat $=90^{\circ}$, causing confusion. As such, we will simply assume that the electric field $\mathbb{E}$ associated with an electron spins at the speed of c/2 (Assumption 2) and in the sequel all the following symbols will refer to an electromagnetic wave of

$$
\begin{gathered}
\lambda v=c=\frac{\omega}{k} \text { with energy } \\
E_{\gamma}=\hbar \omega=\hbar k c=p c \text { and } \omega t=\mathbf{k} \cdot \mathbf{r}=\theta ;
\end{gathered}
$$

within this framework $\mathbb{E}$ spins at a speed of $\omega / 2$, with wave vector $\mathrm{k} / 2$ and the resultant phase angle $\phi$ in

$$
\frac{\omega}{2} t=\frac{\mathbf{k}}{2} \cdot \mathbf{r}=\frac{\theta}{2} \equiv \phi
$$

and the electron has its energy

$$
E=\frac{1}{2} \hbar \omega=\frac{1}{2} E_{\gamma} .
$$

Continuing on our previous thesis (Light, 2012) that the (position) wavefunction $\psi$ has its magnitude proportional to that of the electric field in $\mathrm{B} \subset \mathrm{M}^{[2]}$, we set up (Assumption 3)

$$
\psi(t, x, y, z)=z_{0} e^{-i \cdot \frac{\omega}{2} t}\|E(x, y, z)\|_{\mathbb{C}^{3}}=z_{0} e^{-i \cdot \frac{\omega}{2} t} \cdot \frac{q}{4 \pi \varepsilon_{o}\left(x^{2}+y^{2}+z^{2}\right)},
$$

where $\mathrm{z}_{0} \equiv$ the normalization constant such that 


$$
\begin{aligned}
& z_{0}^{2}=\left(\left.\int_{\mathbb{R}^{3}} \frac{\varepsilon_{o}}{2} \cdot\|E(x, y, z)\|_{\mathbb{C}^{3}}\right|^{2} d x d y d z\right)^{-1}=\left(\frac{1}{2 a} \cdot \frac{q^{2}}{4 \pi \varepsilon_{o}}\right)^{-1} \\
& (\text { see [Feynman, 1963], II-28-1, 2) } \\
& =\left(m c^{2}\right)^{-1} .
\end{aligned}
$$

$\psi$ specifies how $\mathbb{E}$ spins via the phase factor $\exp (-\mathrm{i}(\omega / 2) t)$ (cf. Hannabuss, 1997, for the significance of the phase factor being the defining factor of electromagnetism).

Next we propose the two spin eigenvectors have the following motions, to show that they satisfy the Dirac equation of a free electron:

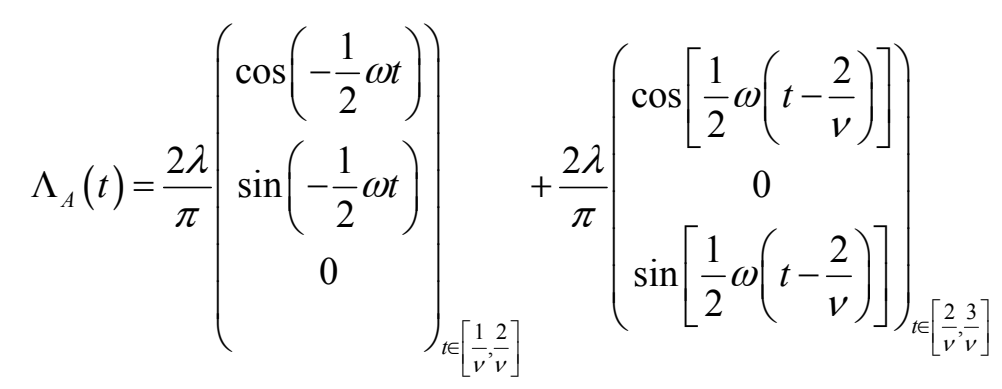

and

$$
\Lambda_{B}(t)=\frac{2 \lambda}{\pi}\left(\begin{array}{c}
\cos \left(-\frac{\pi}{2}-\frac{1}{2} \omega t\right) \\
0 \\
\sin \left(-\frac{\pi}{2}-\frac{1}{2} \omega t\right)
\end{array}\right)_{t \in\left[\frac{1}{v}, \frac{2}{v}\right]}+\frac{2 \lambda}{\pi}\left(\begin{array}{c}
0 \\
\cos \left[-\frac{\pi}{2}-\frac{1}{2} \omega\left(t-\frac{2}{v}\right)\right] \\
\sin \left[-\frac{\pi}{2}-\frac{1}{2} \omega\left(t-\frac{2}{v}\right)\right]
\end{array}\right)_{t \in\left[\frac{2}{v}, \frac{3}{v}\right]} .
$$

The above Equations (2.8), (2.10) and (2.11) imply that

$$
\begin{aligned}
& \frac{\partial \psi}{\partial t}=-\frac{i \omega}{2} \cdot \psi \\
& \frac{\partial \psi}{\partial x}=-\frac{2 x}{x^{2}+y^{2}+z^{2}} \cdot \psi, \text { and thus } \\
& \nabla \psi=-\frac{2 \psi}{x^{2}+y^{2}+z^{2}} \cdot(x, y, z) .
\end{aligned}
$$

Now at $\mathrm{t}=(1 / v)$ we have

$\phi_{A}=-\pi_{(x, y)}$ and $\phi_{B}=-1.5 \pi_{(x, z)}$, so that

$$
\left(\Lambda_{A}\left(\frac{1}{v}\right), \Lambda_{B}\left(\frac{1}{v}\right)\right)=\frac{2 \lambda}{\pi} \cdot\left(\left(\begin{array}{c}
-1 \\
0 \\
0
\end{array}\right),\left(\begin{array}{l}
0 \\
0 \\
1
\end{array}\right)\right),
$$

with 


$$
\begin{aligned}
& \left(\nabla \psi\left(\frac{1}{v}, \Lambda_{A}\left(\frac{1}{v}\right)\right), \nabla \psi\left(\frac{1}{v}, \Lambda_{B}\left(\frac{1}{v}\right)\right)\right)=\left(\left(-\frac{2 \psi}{x}, 0,0\right),\left(0,0,-\frac{2 \psi}{z}\right)\right) \\
& =\psi \cdot\left(\left(\frac{2}{2 \lambda / \pi}, 0,0\right),\left(0,0, \frac{-2}{2 \lambda / \pi}\right)\right)=\psi \cdot\left(\left(\frac{k}{2}, 0,0\right),\left(0,0,-\frac{k}{2}\right)\right) .
\end{aligned}
$$

We next apply a $90^{\circ}$-rotation to each of the above two gradients to obtain the tangent vectors to

$$
\begin{aligned}
& \Lambda_{A}\left(\frac{1}{v}\right) \text { and } \Lambda_{B}\left(\frac{1}{v}\right) \\
& \text { by }-(-i) \nabla \psi,
\end{aligned}
$$

where the outer negative sign will enter the relativistic momentum operator in Equation (2.29) (in analogy with the negative sign in $(-\mathrm{k} \cdot \mathrm{x})$ of $(\omega \mathrm{t}-\mathrm{k} \cdot \mathrm{x}=\theta))$ and the inner negative sign is due to the definition of the momentum operator. That is, we have

$$
\left(\begin{array}{ccc}
0 & -1 & 0 \\
1 & 0 & 0 \\
0 & 0 & 1
\end{array}\right)_{(x, y, z)}\left(\begin{array}{c}
k / 2 \\
0 \\
0
\end{array}\right)=\frac{k}{2}\left(\begin{array}{l}
0 \\
1 \\
0
\end{array}\right)_{(x, y, z)} \text { for } \Lambda_{A}\left(\frac{1}{v}\right)
$$

and

$$
\left(\begin{array}{ccc}
0 & 0 & -1 \\
0 & 1 & 0 \\
1 & 0 & 0
\end{array}\right)_{(x, y, z)}\left(\begin{array}{c}
0 \\
0 \\
-k / 2
\end{array}\right)=\frac{k}{2}\left(\begin{array}{l}
1 \\
0 \\
0
\end{array}\right)_{(x, y, z)} \text { for } \Lambda_{B}\left(\frac{1}{v}\right)
$$

or

$$
-(-i)\left(\nabla \psi\left(\frac{1}{v}, \Lambda_{A}\left(\frac{1}{v}\right)\right), \nabla \psi\left(\frac{1}{v}, \Lambda_{B}\left(\frac{1}{v}\right)\right)\right)=\frac{k}{2}\left(\begin{array}{ll}
0 & 1 \\
1 & 0
\end{array}\right)_{(x, y)} \cdot \psi
$$

where the $\mathrm{z}$-dimension is suppressed.

Similarly, at $\mathrm{t}=(1.5 / \mathrm{v})$, we have

$$
\phi_{A}=-1.5 \pi_{(x, y)} \text { and } \phi_{B}=-2 \pi_{(x, y)} \text {, so that }
$$

$$
\left(\Lambda_{A}\left(\frac{1.5}{v}\right), \Lambda_{B}\left(\frac{1.5}{v}\right)\right)=\frac{2 \lambda}{\pi} \cdot\left(\left(\begin{array}{l}
0 \\
1 \\
0
\end{array}\right),\left(\begin{array}{l}
1 \\
0 \\
0
\end{array}\right)\right),
$$

and

$$
-(-i)\left(\nabla \psi\left(\frac{1.5}{v}, \Lambda_{A}\left(\frac{1.5}{v}\right)\right), \nabla \psi\left(\frac{1.5}{v}, \Lambda_{B}\left(\frac{1.5}{v}\right)\right)\right)=\frac{k}{2}\left(\begin{array}{cc}
1 & 0 \\
0 & -1
\end{array}\right)_{(x, z)} \cdot \psi
$$


Then thirdly at $\mathrm{t}=(2 / \mathrm{v})$, we have

$$
\begin{aligned}
\phi_{A}=-2 \pi_{(x, y)} \text { and } \phi_{B}=-\frac{5}{2} \pi \equiv-\frac{1}{2} \pi_{(x, z)}, \text { so that } \\
\qquad\left(\Lambda_{A}\left(\frac{2}{v}\right), \Lambda_{B}\left(\frac{2}{v}\right)\right)=\frac{2 \lambda}{\pi} \cdot\left(\left(\begin{array}{l}
1 \\
0 \\
0
\end{array}\right),\left(\begin{array}{c}
0 \\
0 \\
-1
\end{array}\right)\right) \text {, and } \\
-(-i)\left(\nabla \psi\left(\frac{2}{v}, \Lambda_{A}\left(\frac{2}{v}\right)\right), \nabla \psi\left(\frac{2}{v}, \Lambda_{B}\left(\frac{2}{v}\right)\right)\right)=\frac{k}{2}\left(\begin{array}{cc}
0 & 0 \\
0 & -1 \\
1 & 0
\end{array}\right)_{(x, y, z)} \cdot \psi \equiv \frac{k}{2}\left(\begin{array}{cc}
0 & -i \\
i & 0
\end{array}\right)_{(x, y)} \cdot \psi
\end{aligned}
$$

by Equation (1.17). To return to their starting positions at $t=(1 / v)$, we continue to move $\Lambda(\mathrm{A})$ and $\Lambda(\mathrm{B})$ to $\mathrm{t}=$ $(2.5 / v)$ and then finally to $t=(3 / v)$ as follows:

At $\mathrm{t}=(2.5 / \mathrm{v})$, we have

$\phi_{A}=\frac{\pi}{2}$ and $\phi_{B}=-\pi_{(y, z)}$, so that

$$
\left(\Lambda_{A}\left(\frac{2.5}{v}\right), \Lambda_{B}\left(\frac{2.5}{v}\right)\right)=\frac{2 \lambda}{\pi} \cdot\left(\left(\begin{array}{l}
0 \\
0 \\
1
\end{array}\right),\left(\begin{array}{c}
0 \\
-1 \\
0
\end{array}\right)\right)
$$

At $\mathrm{t}=(3 / v)$, we finally have

$\phi_{A}=\pi_{(x, z)}$ and $\phi_{B}=-1.5 \pi_{(y, z)}$, so that

$$
\begin{aligned}
& \left(\Lambda_{A}\left(\frac{3}{v}\right), \Lambda_{B}\left(\frac{3}{v}\right)\right)=\frac{2 \lambda}{\pi} \cdot\left(\left(\begin{array}{c}
-1 \\
0 \\
0
\end{array}\right),\left(\begin{array}{l}
0 \\
0 \\
1
\end{array}\right)\right) \\
& =\left(\Lambda_{A}\left(\frac{1}{v}\right), \Lambda_{B}\left(\frac{1}{v}\right)\right) .
\end{aligned}
$$

We now make the following denotation:

$$
\begin{gathered}
P_{t} \equiv i \hbar \frac{\partial}{\partial t}, \quad-P_{x} \equiv i \hbar \frac{\partial}{\partial x}, \quad-P_{y} \equiv i \hbar \frac{\partial}{\partial y}, \quad-P_{z} \equiv i \hbar \frac{\partial}{\partial z}, \quad \text { or }-P_{(x, y, z)} \equiv i \hbar \nabla . \\
\sigma_{1} \equiv \sigma_{(x, y)} \equiv\left(\begin{array}{cc}
0 & 1 \\
1 & 0
\end{array}\right)_{(x, y)}, \sigma_{2} \equiv \sigma_{(x, z)} \equiv\left(\begin{array}{cc}
1 & 0 \\
0 & -1
\end{array}\right)_{(x, z)}, \text { and } \sigma_{3} \equiv \sigma_{(y, z)} \equiv\left(\begin{array}{cc}
0 & -i \\
i & 0
\end{array}\right)_{(x, y)} . \\
\psi_{A} \equiv \psi\left(t, \Lambda_{A}(t)\right) \quad \text { and } \psi_{B} \equiv \psi\left(t, \Lambda_{B}(t)\right) .
\end{gathered}
$$

Then we have by the above Equations (2.12), (2.18), (2.20), (2.22), (2.25), (2.26), and (2.27): 


$$
\sigma_{j}\left(-P_{(x, y, z)}\right)\left(\psi_{A}, \psi_{B}\right)=\frac{\hbar k}{2}\left(\psi_{A}, \psi_{B}\right)
$$

with $\mathrm{j}=1,2,3$ respectively corresponding to $\mathrm{t}=(1 / v),(1.5 / v),(2 / v)$; i.e., we have shown that $\forall \mathrm{j}=1,2,3$,

$$
\left(P_{t}+c \sigma_{j} P_{(x, y, z)}\right)\left(\psi_{A}, \psi_{B}\right)=\left(\frac{\hbar \omega}{2}-\frac{\hbar k c}{2}\right)\left(\psi_{A}, \psi_{B}\right)=(E-p c)\left(\psi_{A}, \psi_{B}\right) .
$$

Since

$$
E^{2}-(p c)^{2}=\left(m_{0} c^{2}\right)^{2}
$$

(where $\mathrm{m}_{0} \equiv$ the rest mass of the electron), if we take the square-root of this equation, then we arrive at the Dirac equation for a free electron,

$$
\left(P_{t}+c \sigma_{j} P_{(x, y, z)}\right)\left(\psi_{A}, \psi_{B}\right)=m_{0} c^{2}\left(\psi_{A}, \psi_{B}\right)
$$

\subsection{Pair-Annihilation}

We now consider the positron, of which Pauli spin matrices carry the opposite signs to those in the above $\sigma_{j}, \mathrm{j}=$ 1,2 , and 3 . Denoting the motions of the two eigenvectors by

$$
\begin{aligned}
& \Lambda_{A}^{\oplus} \text { and } \Lambda_{B}^{\oplus}, \\
& \text { we have } \\
& \Lambda_{A}^{\oplus} \text { move as : } \quad 3 \equiv E \rightarrow 6 \equiv S \rightarrow 9 \equiv W \rightarrow B \rightarrow 3 \equiv E \\
& \text { and } \Lambda_{B}^{\oplus} \text { as }: \quad B \rightarrow 9 \equiv W \rightarrow T \rightarrow 12 \equiv N \rightarrow B .
\end{aligned}
$$

We may thus characterize $\Lambda_{A}^{\oplus}{ }_{A} s$ motion as being restricted to two perpendicularly connected semi-circles with one lying in the Southern plane and the other in the Bottom plane with freedom in the E-W dimension, perhaps identified as "SB." Using the same description, we have $\Lambda_{B}^{\oplus}{ }_{B}$ 's motion in "NW," and back to the electron, we have $\Lambda(\mathrm{A})$ in "NT" and $\Lambda(\mathrm{B})$ in "SE."

To show the pair-annihilation of

$$
\Lambda_{A} \text { with } \Lambda^{\oplus}{ }_{A} \text { and } \Lambda_{B} \text { with } \Lambda_{B}^{\oplus} \text {, }
$$

we first calculate

$$
\begin{gathered}
\Lambda_{A}^{\oplus}(t) \text { and } \Lambda_{B}^{\oplus}(t) \forall t \in\left[\frac{1}{v}, \frac{2}{v}\right] \cup\left[\frac{2}{v}, \frac{3}{v}\right]: \\
\Lambda_{A}^{\oplus}(t)=\frac{2 \lambda}{\pi}\left(\begin{array}{c}
\cos \left[-\frac{1}{2} \omega\left(t-\frac{1}{v}\right)\right] \\
\sin \left[-\frac{1}{2} \omega\left(t-\frac{1}{v}\right)\right] \\
0
\end{array}\right)_{t \in\left[\frac{1}{v}, \frac{2}{v}\right]}+\frac{2 \lambda}{\pi}\left(\begin{array}{c}
\cos \left[\pi+\frac{1}{2} \omega\left(t-\frac{2}{v}\right)\right] \\
0 \\
\left.\left.\sin \left[\pi+\frac{1}{2} \omega\left(t-\frac{2}{v}\right)\right]\right)_{t \in\left[\frac{2}{v}, \frac{3}{v}\right.}\right]
\end{array},\right.
\end{gathered}
$$

and 


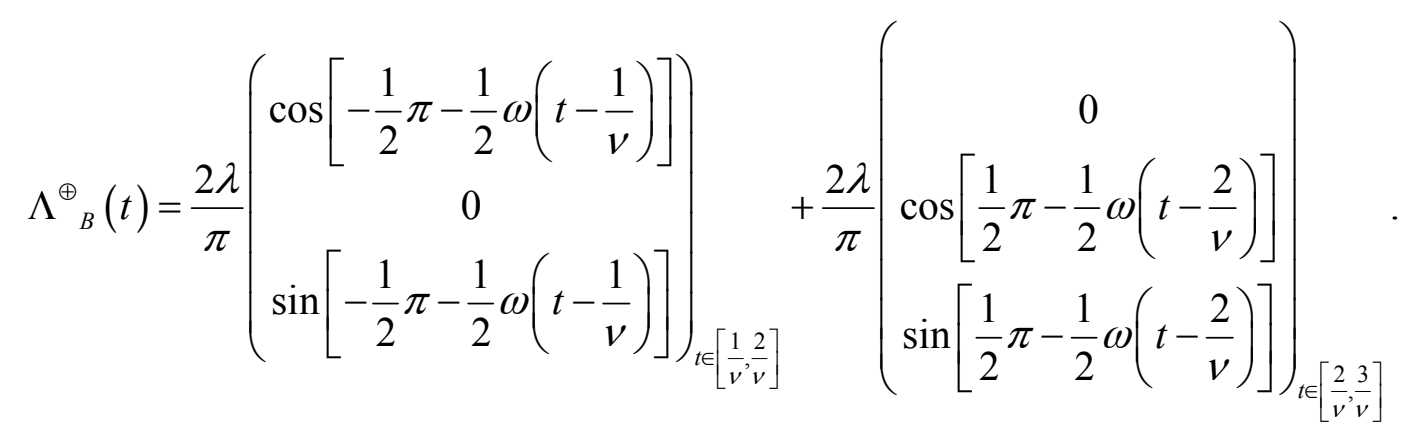

Then we have

$$
\begin{aligned}
& \forall t \in\left[\frac{1}{v}, \frac{2}{v}\right] \frac{\pi}{2 \lambda}\left(\Lambda_{A}(t)+\Lambda_{A}^{\oplus}(t)\right)=\cos \left(-\frac{1}{2} \omega t\right)+\cos \left[-\frac{1}{2} \omega\left(t-\frac{1}{v}\right)\right] \\
& =2 \cos \frac{1}{2}\left(-\omega t+\frac{1}{2} \omega \cdot \frac{1}{v}\right) \cos \frac{1}{2}\left(-\frac{1}{2} \omega \cdot \frac{1}{v}\right) \\
& =2 \cos \frac{1}{2}(-\omega t+\pi) \cos \left(-\frac{1}{2} \pi\right)=0,
\end{aligned}
$$

and for the sine branch,

$$
\begin{aligned}
& \sin \left(-\frac{1}{2} \omega t\right)+\sin \left[-\frac{1}{2} \omega\left(t-\frac{1}{v}\right)\right] \\
& =2 \sin \frac{1}{2}\left(-\omega t+\frac{1}{2} \omega \cdot \frac{1}{v}\right) \cos \frac{1}{2}\left(-\frac{1}{2} \omega \cdot \frac{1}{v}\right)=0,
\end{aligned}
$$

implying that the combined electric field shrinks to zero, i.e., a disappearance of both the electron and the positron.

For bookkeeping completeness we next calculate

$\Lambda_{A}(t)+\Lambda_{A}^{\oplus}(t) \forall t \in\left[\frac{2}{v}, \frac{3}{v}\right] ;$ here we have:

$$
\begin{aligned}
& \cos \left(\frac{1}{2} \omega\left(t-\frac{2}{v}\right)\right)+\cos \left[\pi+\frac{1}{2} \omega\left(t-\frac{2}{v}\right)\right] \\
& =2 \cos \frac{1}{2}\left(\pi+\omega\left(t-\frac{2}{v}\right)\right) \cos \frac{1}{2}(-\pi)=0,
\end{aligned}
$$

with the sine branch sharing the same factor of $\cos \frac{1}{2}(-\pi)=0$.

Likewise for 


$$
\begin{aligned}
& \Lambda_{B}(t)+\Lambda_{B}^{\oplus}(t): \\
& (1) \forall t \in\left[\frac{1}{v}, \frac{2}{v}\right] \text { we have } \\
& \cos \left(-\frac{\pi}{2}-\frac{1}{2} \omega t\right)+\cos \left(-\frac{\pi}{2}-\frac{1}{2} \omega\left(t-\frac{1}{v}\right)\right) \\
& =2 \cos \frac{1}{2}\left(-\pi-\omega t+\frac{1}{2} \cdot \frac{\omega}{v}\right) \cos \frac{1}{2}\left(-\frac{1}{2} \frac{\omega}{v}\right)=0 \\
& (=\text { the sum of the two sine functions, also); } \\
& (2) \forall t \in\left[\frac{2}{v}, \frac{3}{v}\right] \text { we have } \\
& \cos \left(-\frac{\pi}{2}-\frac{1}{2} \omega\left(t-\frac{2}{v}\right)\right)+\cos \left(\frac{\pi}{2}-\frac{1}{2} \omega\left(t-\frac{2}{v}\right)\right) \\
& =2 \cos \frac{1}{2}\left(-\omega\left(t-\frac{2}{v}\right)\right) \cos \frac{1}{2}(-\pi)=0 \\
& \text { (= the sum of the two sine functions, also). }
\end{aligned}
$$

\section{Summary Remarks}

First, the two eigenstates $\psi(\mathrm{A})$ and $\psi(\mathrm{B})$ in the superposition of

$$
\left\{\psi=c_{1} \psi_{A}+c_{2} \psi_{B} \mid c_{1}, c_{2} \in \mathbb{Q}\right\}
$$

are either/or,but not both (Shankar, 1994, for the meaning of quantum superpositions), so that $\Lambda(\mathrm{A}(\mathrm{t}))$ alone specifies the spinning motion of the electric field $\mathbb{E}(\mathrm{t})$, i.e.,

$$
9 \rightarrow 12 \rightarrow 3 \rightarrow T \rightarrow 9
$$

In details, from $t=(1 / v)$ to $(2 / v)$, while to a photon it would be a $2 \pi$-cycle due to the time duration of $\Delta t=(1 / v)$, yet to the electron it is only half a cycle - - i.e., from $9 \equiv \mathrm{W}$ to $3 \equiv \mathrm{E}$, displaying itself with an energy of $\mathrm{E}=(1 / 2) \hbar \propto$ at $3 \equiv \mathrm{E}$. Then from $\mathrm{t}=(2 / v)$ to $(3 / v)$ the electron completes its $2 \pi$-cycle by returning from $3 \equiv \mathrm{E}$ to $9 \equiv \mathrm{W}$ (although via a different plane and again revealing itself with an energy $E=(1 / 2) \hbar \circ$ at $9 \equiv W)$. Note also that it takes

$$
9 \rightarrow 12 \rightarrow 3 \rightarrow T \rightarrow 9 \rightarrow 12 \rightarrow 3 \rightarrow T \rightarrow 9
$$

for the electron to return to the original reference frame of $(x, y, z)$; this is caused by the turn at $3 \equiv E$ that changes to a different plane of

$$
3 \rightarrow T \rightarrow 9 \text { from that of } 9 \rightarrow 12 \rightarrow 3
$$

Second, since the momentum operator is a linear operator, any linear combination at $\mathrm{t}=0$ of some three energy density points $\beta(0)=(0,1,0) \equiv \mathrm{N} \equiv 12, \alpha(0)=(1,0,0) \equiv \mathrm{E} \equiv 3$ and $\gamma(0)=(0,0,1) \equiv \mathrm{T}$ with coordinates $(\mathrm{a}, \mathrm{b}, \mathrm{c})$ has $\forall \mathrm{t}$ the path

$$
a \alpha(t)+b \beta(t)+c \gamma(t)
$$

satisfy the Dirac equation. I.e., the electric field has a rigid spinning motion that is determined by $\Lambda(\mathrm{A}(\mathrm{t}))$ :

$$
9 \rightarrow 12 \rightarrow 3 \rightarrow T \rightarrow 9 .
$$

Third, in the above motion of $\Lambda(\mathrm{A}(\mathrm{t}))$, the fact that the spinning position through $3 \equiv \mathrm{E}$ can be identified with $\Lambda(\mathrm{B})$ is only because $3 \equiv \mathrm{E}$ happens to lie in the path of $\Lambda(\mathrm{B}(\mathrm{t}))$ : 


$$
T \rightarrow 3 \rightarrow B \rightarrow 6 \rightarrow T \text {. }
$$

$\Lambda(\mathrm{B}(\mathrm{t}))$ can be transformed from $\Lambda(\mathrm{A}(\mathrm{t}))$ by a change of frame through a $90^{\circ}$-counterclockwise-turn followed by a $180^{\circ}$-turn on a perpendicular plane.

Fourth, we conjecture that the set of Pauli matrices is the source of charge of an electron for the following two reasons: (1) Reversing the signs of $\sigma(\mathrm{j})$ as associated with the electron turn it into a positron.

(2) The matrix

$$
\left(\begin{array}{cc}
0 & -i \\
i & 0
\end{array}\right)
$$

changes the planes of rotation and the intersection of the two semi-circles presents a singularity (not differentiable). We thus surmise that it is at the two turning points of $9 \equiv \mathrm{W}$ and $3 \equiv \mathrm{E}$ that virtual photons transmit charges.

Fifth, following our previous analysis that the wave universe $\mathrm{B}$ is a black hole in $\mathrm{M}^{[2]}$, we see the possibility that $\mathrm{B}$ as a whole is spinning (Longo, 2011) and this cosmic spin happens to favor the set of Pauli matrices of our ordinary matter in our environment.

Sixth, to make a smooth continuation from our previous work to this present study, we repeat that the (electron, wave) carries a total energy of $\mathrm{E}=(3 / 4) \mathrm{E}+(1 / 4) \mathrm{E}$, with the two component energies respectively contained in $\mathfrak{M}^{[1]}$ x B (where the ratio of 3:1 came from Feynman's analysis of the electromagnetic mass, II-28-3). An electron can disappear, within a time duration of $(h / \mathrm{E})$, from $\mathfrak{M}^{[1]}$ and exists completely as the above wave $\psi$ in $\mathrm{B} \subset \mathfrak{M}^{[2]}$ carrying the entire energy $\mathrm{E}$, which equals 1.6 times the laboratory measured energy of the electron (Light, 2012).

In closing, guided by Pauli matrices and the Dirac equation, we are able to, in this paper, specify the graph of the diffeomorphism

$$
\begin{aligned}
& \boldsymbol{h}: \boldsymbol{M}^{[1]} \rightarrow \mathbf{B} \subset M^{[2]} \text { to be } \\
& M^{[3]}=\left\{(t+i t, x+i y, y+i z, z+i x) \mid(t, x, y, z) \in \mathbb{R}^{1+3}\right\} .
\end{aligned}
$$

With waves of energies specified in the above coordinates of a combined spacetime 4-manifold $\mathrm{M}^{[3]}$ instead of abstract probabilities rooted in vacuum, we will pursue further details of the quantum geometry to contribute to the research community, such as a reduction of the dimensionalities in the quantum information systems.

\section{References}

Sven, A., Heiko, B., Christoph, H. K., \& Carsten, M. (2012). Spin dynamics in the Kapitza-Dirac effect, Physical Review Letters 109, 043601. http://dx.doi.org/10.1103/PhysRevLett.109.043601

Barut, O., \& Bracken, A. J. (1981). Zitterbewegung and the internal geometry of the electron. Physical Review D 23, 2454-2463. http://dx.doi.org/10.1103/PhysRevD.23.2454

Costa, L. F., Herdeiro, C., Natário, J., \& Zilhão, M. (2012). Mathisson's helical motions for a spinning particle: are they unphysical? Physical Review D, 85, 024001 (11 pages). http://dx.doi.org/10.1103/PhysRevD.85.024001

Demikhovskii, V. Ya., Maksimova, G. M., Perov, A. A., \& Frolova, E. V. (2010). Space-time evolution of Dirac wave packets. Physical Review A, 82, 052115. http://dx.doi.org/10.1103/PhysRevA.82.052115

Dreisow, F., Longhi, S., Nolte, S., Tünnermann, A., \& Szameit, A. (2012). Vacuum instability and pair production in an optical setting. Physical Review Letters, 109, 110401. http://dx.doi.org/10.1103/PhysRevLett.109.110401

Feynman, R. P. et al. (1963). The Feynman lectures on physics. Reading: Addison-Wesley.

Hannabuss, K. (1997). An introduction to quantum theory. Oxford: Oxford University Press.

Light, G. L. (2012). A heuristic derivation of a Planck-Heisenberg energy formula. Applied Physics Research, 4, 138-145. http://dx.doi.org/10.5539/apr.v4n1p138

Longo, M. J. (2011). Detection of a dipole in the handedness of spiral galaxies with redshifts. Physics Letters B, 
699(4), 224-229. http://dx.doi.org/10.1016/j.physletb.2011.04.008

Rau, A. R. P. (2009). Mapping two-qubit operators onto projective geometries. Physical Review A, 79, 042323. http://dx.doi.org/10.1103/PhysRevA.79.042323

Shankar, R. (1994). Principles of quantum mechanics. New York: Spinger.

Witten, E. (1981). A new proof of the positive energy theorem. Communications in Mathematical Physics, 80, 381-402. http://dx.doi.org/10.1007/BF01208277 